gr-qc/9302010

\title{
Cosmology with Nonminimal Derivative Couplings
}

\author{
LUCA AMENDOLA \\ Osservatorio Astronomico di Roma \\ Viale del Parco Mellini, 84 \\ I-00136 Rome, Italy
}

\begin{abstract}
We study a theory which generalizes the nonminimal coupling of matter to gravity by including derivative couplings. This leads to several interesting new dynamical phenomena in cosmology. In particular, the range of parameters in which inflationary attractors exist is greatly expanded. We also numerically integrate the field equations and draw the phase space of the model in second order approximation. The model introduced here may display different inflationary epochs, generating a non-scale-invariant fluctuation spectrum without the need of two or more fields. Finally, we comment on the bubble spectrum arising during a first-order phase transition occurring in our model.
\end{abstract}

\section{Introduction}

Scalar fields in General Relativity has been a topic of great interest in the latest years, mainly because the scalar field dynamics allows to investigate the detailed features of the early Universe. Without the need of a specific equation of state, a Universe filled by a phenomenological scalar field leads to an accelerated phase of expansion, the inflation, in almost any kind of self-interaction potential. As it has been shown in chaotic models of inflation 1 , the accelerated phase, either power-law 2 or quasi-exponential, is a phase-space attractor for most of the initial conditions, and this result also extends to a large class of inhomogeneous and anisotropic space-times $\mathrm{B}$. The class of successful models has been enlarged to scalar fields with a nonminimal coupling (NMC) to the curvature scalar $R$ in the gravity Lagrangiant, commonly in the form of a term $\sqrt{-g} f(\phi) R$, where $f(\phi)$ is a function of the scalar field $\phi$. The motivations for this step are manyfold: the idea that the fundamental constants are not constant, the "machian" theory of gravitation embodied in the Jordan-Brans-Dicke theoryE, the renormalizing term arising in quantum field theory in curved space , the possibility to have gravity as a spontaneous symmetry-breaking effect the Kaluza-Klein compactification scheme 8 , the low-energy limit of the superstring theory $\theta^{2}$. Moreover, the NMC term has been employed to produce an oscillating Universe, 10 to reconcile cosmic strings production with inflation,11 to generate a modified Newtonian dynamics able 
to model flat rotation curves in galaxies.12 Last but not least, an NMC term allows to solve the "graceful exit" problem of the old inflation by slowing the false-vacuum expansion, as in extended inflation 13. Recently, the NMC models have been generalized in various directions: models with different coupling functions 14 , with generalized coupling to the inflaton sector 5 , with fourth-order gravity 16 , and with a coupling to a dark matter sector 17 .

In this Letter we wish to further explore the influence of nonminimal couplings in cosmology by introducing the nonminimal derivative coupling (NMDC) to gravity. In this class of models, the coupling function is also function of the derivatives of $\phi: f=f\left(\phi, \phi_{; \mu}, \phi_{; \mu \nu}, \ldots\right)$. Derivative couplings, although rares, are not a novelty in physics: the field theory of scalar quantum electro-dynamics includes derivative couplings between the electro-magnetic vector $A_{\mu}$ and the scalar field $\phi$; this kind of interaction is indeed required by the $U(1)$ gaugeinvariance of the theory. In addition, if the idea underlying the nonminimal coupling theory is that the Newton constant $G$ itself depends on the gravitational field source mass, it seems more natural to couple the curvature to the energy-momentum tensor of the matter, introducing the terms $T R$ and $T_{\mu \nu} R^{\mu \nu}$, which in fact contain derivative couplings. The NMDC model can be thought of as the scalar field formulation of the hypothesis that $G=G(\rho)$, where $\rho$ is the energy density of the gravitational field source.

One of the aims of this Letter is to see whether the presence of NMDCs allows to find inflationary attractors in models where otherwise they are not present. We studied a large class of NMC models in Ref. (18). For future reference, let us summarize the main results of Ref. (18). Hereinafter, we will use $f=f(\phi)$ referring to the non-derivative terms coupled to the curvature scalar, and $f_{1}=f_{1}\left(\phi, \phi_{; \alpha}\right)$ when we refer to all the terms, including the derivative terms, coupled to the curvature scalar. In Ref. (18) the coupling function $f(\phi)$ is left as general as possible, and the potential is assumed to be $V=\lambda f^{M}$. It is also assumed that $f(\phi)$ is semi-positive definite and that, for large $|\phi|$, it grows monotonically faster than $\phi^{2}$. The case $f \sim \phi^{2}$ is the standard choice, and has been extensively treated

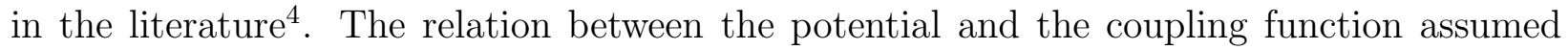
in Ref. (18) allows to determine the class of models with successful inflationary attractors; here "successful" means that the inflation eventually stops and a Friedmann stage begins. In Ref. (18) and in the present work the inflationary attractors are all asymptotic, in the limit of large $|\phi|$; in this limit the influence of the coupling is clearly larger. Moreover, the spirit of chaotic inflation is that whatever the initial conditions of the Universe are, the dynamical trajectory falls onto an inflationary attractor. This is verified only if the attractor itself extends to very large values of the field variables, until the classical description loses meaning (i.e. near the Planck boundary). In Ref. (18) it is shown that such asymptotic attractors exist only if $2 \leq M<2+\sqrt{3}$, whatever functional form of $f(\phi)$ is considered. In this range, it is found for the cosmic scale factor $a(t)$ a power-law inflation $a \sim t^{p}$ with exponent

$$
p=\frac{3+(M-2)^{3}}{(M-2)^{2}(M-1)} .
$$

In the conformally rescaled frame the power-law exponent has a much simpler form, $\tilde{p}=$ $3 /(M-2)^{2}$. If $M=2$ a deSitter exponential inflation is found. Further, in the cases in which a successful inflation exists, the spectrum of primordial fluctuations is independent of 
$f(\phi)$ and depends on the wavenumber $k$ according to the power-law $k^{1 /(1-\tilde{p})}$. Finally, in Ref. (18) it is shown that the fluctuations have a Gaussian distribution. In the present work we extend some of the cited results to derivative couplings. We assume as in Ref. (18) that $V=\lambda f^{M}$, but now we will freeze the functional form of $f(\phi)$ to a power-law, $f(\phi)=\phi^{2 m}$. We will show that the narrow range in which the parameter $M$ is confined in NMC models is greatly expanded when one includes a derivative coupling.

It rises natural the question of whether the conformal transformation 19 , which allows one to put complicated unconventional gravity theory in pure Einsteinian form with one or more scalar fields, works also when derivative couplings are present. We will show schematically that a conformal rescaling $\tilde{g}_{\mu \nu}=e^{2 \omega} g_{\mu \nu}$ cannot recast our theory in Einsteinian form. In order to recover the Einstein field equations the metric transformation should be generalized to a Legendre transformation, as in Ref. (20). This would introduce in the equations additional tensor fields, instead of additional scalar fields as in non-derivative cases. Due to this reason, we avoided here the use of metric transformations.

The plan of this paper is as follows: in the next section we introduce the derivative couplings, in Sect. 3 we derive the equations of motion for the fields, in Sect. 4 we perform the second-order approximation, in Sect. 5 we discuss the phase space of some models and finally in Sect. 6 we present the conclusions.

\section{Derivative couplings}

Terms with NMDCs generalize the Einstein-Hilbert Lagrangian. The most general gravity Lagrangian linear in the curvature scalar $R$, quadratic in $\phi$, containing terms with four derivatives includes all of the following terms:

$$
\begin{aligned}
& L_{1}=\mu \phi_{; \alpha} \phi^{; \alpha} R ; \quad L_{2}=\tau \phi^{; \alpha} \phi^{; \beta} R_{\alpha \beta} ; \quad L_{3}=\eta \phi \square \phi R ; \\
& L_{4}=\theta \phi \phi^{; \alpha \beta} R_{\alpha \beta} ; \quad L_{5}=\nu \phi \phi_{; \alpha} R^{; \alpha} ; \quad L_{6}=\sigma \phi^{2} \square R .
\end{aligned}
$$

The constants $\mu, \tau, \ldots$ have the same dimensions in natural units as the Newton constant $G$, namely mass $^{-2}$. Here and in the following the conventions are: signature $(+---)$; units $8 \pi G=c=1$. Due to the following relations

$$
\begin{aligned}
0 & =\int d^{4} x \sqrt{-g} \square\left(\phi^{2} R\right)=\int d^{4} x \sqrt{-g}\left[2 \phi \square \phi R+2 \phi_{; \alpha} \phi^{; \alpha} R+\phi^{2} \square R\right], \\
0 & =\int d^{4} x \sqrt{-g} \nabla_{\mu}\left(R \nabla^{\mu} \phi^{2}\right)=\int d^{4} x \sqrt{-g}\left[2 \phi \phi^{; \alpha} R_{; \alpha}+2 \phi \square \phi R+2 \phi_{; \alpha} \phi^{; \alpha} R\right], \\
0 & =\int d^{4} x \sqrt{-g} \nabla_{\mu}\left(\phi \phi_{; \nu} R^{\mu \nu}\right)=\int d^{4} x \sqrt{-g}\left[\phi_{; \mu} \phi_{; \nu} R^{\mu \nu}+\phi \phi_{; \mu \nu} R^{\mu \nu}+\phi \phi_{; \nu} R_{; \mu}^{\mu \nu}\right],
\end{aligned}
$$

three of the six terms $L_{1}-L_{6}$ can be neglected (also taking into account the Bianchi identities). Other total divergences, like $\left(\phi^{2} R_{; \nu}^{\mu \nu}\right)_{; \mu}$ and $\left(\phi^{2} R^{\mu \nu}\right)_{; \mu \nu}$ are linear combinations of Eqs. (3). Nevertheless, the equations remain hopelessly complicated. We take here a first step. We keep only the derivative term $L_{1}$, together with the NMC correction $L_{0}=\xi f(\phi) R$. This particular choice turns out to be the simplest one. Moreover, the terms studied here are those appearing in the simplest "natural" coupling, namely $T R$. In this way we may 
draw an useful comparison between Ref. (18), where $L_{0}$ was the only correction to Einstein gravity, and the present work. We are then left with the following Lagrangian density

$$
\begin{aligned}
L & =-R+f_{1}\left(\phi, \phi_{; \alpha}\right) R+\phi_{; \alpha} \phi^{; \alpha}-2 V(\phi), \\
f_{1}\left(\phi, \phi_{; \alpha}\right) & =\xi f(\phi)+\mu \phi_{; \alpha} \phi^{; \alpha} .
\end{aligned}
$$

\section{$3 \quad$ Field equations}

The set of equations derived from (4) is

$$
\begin{aligned}
G_{\mu \nu}\left(1-f_{1}\right) & =g_{\mu \nu} \square f_{1}-f_{1 ; \mu \nu}+\mu \phi_{; \mu} \phi_{; \nu} R+T_{\mu \nu} \\
T_{\mu \nu} & =\phi_{; \mu} \phi_{; \nu}-\frac{1}{2} g_{\mu \nu} \phi_{; \alpha} \phi^{; \alpha}+V(\phi) g_{\mu \nu}
\end{aligned}
$$

while the scalar field equation is

$$
\square \phi(1+\mu R)+\mu \phi_{; \alpha} R^{; \alpha}-\xi f^{\prime} R / 2+V^{\prime}=0 .
$$

It can already be seen that, even if the new terms introduced by the derivative coupling (let us call them $\mu$-terms) do not determine the critical points of the theory, they do contribute to the asymptotic dynamical properties of Eq. (6). Indeed we will see that in the limit of large $R$ and $R_{; \alpha}$ (in other words, in the early Universe), the $\mu$-terms will sensibly modify the behavior of the non-derivative theory.

The trace of Eqs. (5) reads simply

$$
R(\xi f-1)=T+3 \square f_{1},
$$

where $T=-\phi_{; \alpha} \phi^{; \alpha}+4 V$. The derivative of the Ricci scalar, to be used in Eq. (6), is

$$
R_{; \alpha}=\frac{1}{\xi f-1}\left[T_{; \alpha}+3 \square f_{1, \alpha}-\xi f^{\prime} \phi_{; \alpha} R\right] .
$$

In a Friedmann-Robertson-Walker (FRW) spatially flat metric with scale factor $a(t)$, the $(0,0)$ component of (5) is

$$
H^{2}\left(1-f_{1}\right)=H \dot{f}_{1}+\frac{\mu}{3} R \dot{\phi}^{2}+\frac{1}{3}\left(\frac{1}{2} \dot{\phi}^{2}+V\right)
$$

where $H=\dot{a} / a$ and where our conventions are such that $R=-6 \dot{H}-12 H^{2}$. From now on, we confine ourselves to a FRW spatially flat metric. The system (6-9) is closed. We have five degrees of freedom $H, \phi, \dot{\phi}, \ddot{\phi}, \phi^{I I I}$ and indeed we have one equations of fourth order in $\phi[$ Eq. (6)] and one of first order in $H$ [Eq. (9)]. The solution of this system appears very difficult. In the next section we will make use of a second-order approximation to derive the asymptotic properties of our model and to sketch its phase space.

Let us conclude this section showing how a conformal transformation operates on the NMDC theory. Ordinary NMC theories are recast in Einstein form by the metric rescaling 19 $\tilde{g}_{\mu \nu}=e^{2 \omega} g_{\mu \nu}$, with

$$
e^{2 \omega}=|\partial L / \partial R| .
$$


Adopting the same formula, in our case we get $e^{2 \omega}=\left|1-f_{1}\right|$. Now, we have that the Einstein tensor $G_{\mu \nu}=R_{\mu \nu}-R g_{\mu \nu} / 2$ trasforms according to

$$
G_{\mu \nu}=\tilde{G}_{\mu \nu}-2\left[\omega_{; \mu} \omega_{; \nu}+\frac{1}{2} g_{\mu \nu} \omega_{; \alpha} \omega^{; \alpha}+g_{\mu \nu} \square \omega-\omega_{; \mu \nu}\right] .
$$

Inserting (11) into (5), with the choice (10), one has

$$
\tilde{G}_{\mu \nu}=3 e^{-4 \omega}\left(f_{1 ; \mu} f_{1 ; \nu}-\frac{1}{2} g_{\mu \nu} f_{1 ; \alpha} f_{1}^{; \alpha}\right)+\mu e^{-2 \omega} \phi_{; \mu} \phi_{; \nu} R+e^{-2 \omega} T_{\mu \nu},
$$

which is not in Einsteinian form. Any other choice of the conformal factor would not cancel the second derivatives of $f_{1}$ which are present in (5). Then, the right-hand-side of (12) could not be written as a scalar field energy-momentum tensor. Notice that $R$ at the right-handside is not trasformed. If one also trasforms $R$ in (12), additional terms like $(\square \omega) \phi_{; \mu} \phi_{; \nu}$ arise.

\section{Second-order approximation}

In slow-rolling motion one neglects all terms with more than one time derivative in the field equations. In this section we are more general, and we examine the field equations by neglecting all terms of order higher than the second one. This gives us three advantages: the equations are notably simplified, we can calculate analytically some inflationary solutions, and we can plot the phase-space portrait of the model. Neglecting all terms higher than second order in (6) we have

$$
\square \phi\left(1+\mu R^{(0)}\right)+\mu \dot{\phi} \dot{R}^{(1)}-\xi f^{\prime} R^{(2)} / 2+V^{\prime}=0 .
$$

The indexes in parentheses denote the order to which $R$ and $\dot{R}$ are to be calculated. From Eqs. (7,8) and for $\xi f \gg 1$ we have

$$
\begin{aligned}
R^{(0)} & =\frac{4 V}{\xi f}, \\
R^{(2)} & \left.=\frac{1}{\xi f}\left[-\dot{\phi}^{2}+4 V+3 \xi \square f\right)\right], \\
\dot{R}^{(1)} & =\frac{4 \dot{\phi}}{\xi f^{2}}\left[V^{\prime} f-V f^{\prime}\right] .
\end{aligned}
$$

We now explicitely adopt the functional relation $V=\lambda f^{M}$. This will simplify our equations. Eq. (13) then writes

$$
\begin{aligned}
& \square \phi\left[f+\frac{4 \mu \lambda}{\xi} f^{M}-\frac{3}{2} \xi f^{\prime 2}\right]+f^{\prime} \dot{\phi}^{2}\left[\frac{4 \mu \lambda}{\xi} f^{M-1}(M-1)+\frac{1}{2}\left(1-3 \xi f^{\prime \prime}\right)\right] \\
& +\lambda f^{\prime} f^{M}(M-2)=0 .
\end{aligned}
$$

Let us call $A$ the coefficient of $\square \phi$ and $B$ the coefficient of $f^{\prime} \dot{\phi}^{2}$. In order to avoid singularities in Eq. (17) one should have a nonvanishing coefficient $A$ for $\xi f \gg 1$ (in the opposite limit 
the equations reduce to the minimally coupled model and the regularity follows automatically). A sufficient condition, although not a necessary one, is that $\xi, \mu \leq 0$, along with the already imposed condition $f \geq 0$. This assumption greatly simplifies the investigation of the dynamical properties of the model under study and will be adopted in the following. It also makes clearer the comparison with previous work in ordinary NMC theories, where the condition $\xi<0$ is often adopted to get a regular metric rescaling. Notice that $\xi, \mu \leq 0$ ensures the positivity of the effective Newton constant in the Lagrangian density (雨).

The d'Alambertian operator reads $\square \phi=\ddot{\phi}+3 H \dot{\phi}$ in FRW metric, where the Hubble function $H$ is to be taken from Eq. (9) (to the first order):

$$
\xi H^{2} f+\xi H \dot{f}+\frac{4 \mu V}{3 \xi f} \dot{\phi}^{2}+\frac{1}{3}\left(\frac{1}{2} \dot{\phi}^{2}+V\right)=0 .
$$

The asymptotic behavior of Eq. (17) can be now easily discussed in the limit in which some of the terms in $A$ and $B$ can be neglected. A first consideration is however immediate: for $M<2$ the second derivative of the effective potential in Eq. (17) is negative, in the hypothesis that $f$ grows monotonically with $|\phi|$. As a consequence, any attractor solution of Eq. (17) leads to ever growing $f$; the Friedmann behavior cannot be reached. Let us make an example. If we assume $f(\phi) \rightarrow \phi^{2 m}$ for large $|\phi|$, it turns out that if $M<2-1 / m$ one has that $f^{\prime 2}$ dominates in $A$ and $f^{\prime \prime}$ in $B$. Then Eq. (17) simplifies to

$$
\square f-[2 \lambda(M-2) / 3 \xi] f^{M}=0
$$

(where $\square f=f^{\prime} \square \phi+f^{\prime \prime} \dot{\phi}^{2}$ ). In this case the influence of the $\mu$-terms disappears; the model reduces to the ordinary NMC theory, already discussed in Ref. (18). There it was found that for $M<2$ no successful inflation was allowed. Indeed, in Eq. (19) the effective potential derivative is negative definite (since $\xi<0$ and $M<2$ ). As already discussed, the attractor solutions of Eq. (19) are directed toward increasing $f$; the Friedmann behavior will never be recovered. The same conclusion holds for any $f(\phi)$, provided that $f^{\prime 2} \gg f^{M}, f$.

In the special case $M=2$ one sees that $\ddot{\phi}=\dot{\phi}=0$ is a solution of (17). One has then an asymptotic deSitter inflation, analogously to what occurs in NMC models. We will not give details of this case, since the dynamics depends on the behavior of $V$ and $f$ at small $\phi$, while here we are interested mainly in the asymptotic properties.

Let us come to the third, more interesting, case, namely $M>2$. Now $f^{M}$ dominates in $A$ and $f^{M-1}$ in $B$. Eq. (17) then reads

$$
\square \phi+\frac{f^{\prime}}{f} \dot{\phi}^{2}(M-1)+\frac{\xi}{4 \mu} f^{\prime}(M-2)=0 .
$$

It worths remarking that the effective potential is $U(\phi)=\xi(M-2)(4 \mu)^{-1} f(\phi)$; the coupling function $f(\phi)$ reveals itself as an effective potential for the theory. Notice also that, contrary to the previous case, the constant $\mu$ plays here an important role. Let us put now $f(\phi)=x^{m}$, where $x \equiv \phi^{2}$. It then follows

$$
\square x-\frac{\dot{x}^{2}}{2 x}[1-2 N-2 m]+\frac{\xi N}{\mu} x^{m}=0,
$$


where $N=m(M-2)$, for $x \geq 0$. To have a positive-definite potential derivative is sufficient to have $M>2$ (provided that $\xi / \mu>0$ ).

To find explicit solutions to Eq. (21) we need also $H=H(\phi, \dot{\phi})$. From Eq. (18), neglecting all terms higher than first order and in the limit $\xi f \gg 1$, we get for $M>2-1 / m$ (i.e. $N>-1$ )

$$
\left[H^{(1)}\right]^{2}=\frac{\lambda}{3|\xi|} x^{N-1}\left[\frac{3 \mu}{\xi} \dot{x}^{2}+x^{m+1}\right] .
$$

Let us put a trial solution $\dot{x}=b x^{p}$ in (21) and (22). In the limit of large $x$ we find the attractor

$$
\begin{aligned}
p & =(m-N) / 2, \\
b & =-\frac{N}{\sqrt{3}}\left(\frac{\xi^{3}}{\lambda \mu}\right)^{1 / 2} .
\end{aligned}
$$

It can be easily proved that this really is an attractor; a graphic evidence is provided by our phase-space portraits, Figs (11.21). Along the solution (23) the term $\ddot{x}$ is negligible; this justifies the second order approximation previously performed. Integrating $\dot{x}=b x^{p}$ one gets

$$
x=x_{0}(1+t / \tau)^{1 /(1-p)},
$$

where $\tau^{-1}=b(1-p) x_{0}^{p-1}$ and $x(t=0)=x_{0}$. For $p<1$ the time constant $\tau$ is negative; clearly our approximations break down for $t \rightarrow \tau$. The inflationary regime we will find also breaks for $t \rightarrow \tau$. Along the attractor we have the Hubble function

$$
H=(\lambda / 3|\xi|)^{1 / 2} x^{(N+m) / 2},
$$

and the cosmic scale factor

$$
a(t)=a_{0} \exp \left\{E\left[1-(1+t / \tau)^{k}\right]\right\},
$$

where

$$
E=\frac{3(|\mu / \xi|) x_{0}^{N+1}}{N(N+1)}, \quad k=\frac{2(N+1)}{2+N-m} .
$$

The scale factor expansion is always inflationary for $t<\tau$. For $t \ll \tau$ the expansion follows the deSitter law $a \sim \exp [(E|k / \tau|) t],(k / \tau$ is always negative). The constant $E$ sets the total number of $e$-foldings of the inflationary stage. If, for instance, $\xi$ and $\mu$ are approximatively equal, values of $\phi_{0}=x_{0}^{1 / 2}$ around unity in Planck units are required to have $E>60$, the same requirement commonly found in chaotic inflation. This kind of behavior is often called quasideSitter inflation. The narrow range in which viable inflation is found in NMC theories 18 , $2 \leq M<2+\sqrt{3}$, is here expanded to $M \geq 2$, without upper bound. The effect of including a derivative coupling is then to substantially enlarge the class of viable inflationary models. This is the main result of this paper. 
It can be checked that $H(t)$ and $x(t)$ decrease as the time increases. As $|\phi|$ decreases, the solution crosses to the NMC region, where the derivative coupling is negligible compared to the non-derivative one. In particular, the attractor enters the NMC region for

$$
x<x_{1}=\left[\frac{3 \xi^{2} m^{2}}{2|\mu| \lambda}\right]^{1 /(N+1)} .
$$

As $|\phi|$ further decreases, $\xi f(\phi)$ becomes smaller than unity for $x<x_{2}=\xi^{-1 / m}$ and the attractor enters the minimally coupled central region. For instance, in a model in which $|\mu| \lambda=3 / 2$ and $M=3, m=1$, one has $x_{1}=\xi$ and $x_{2}=1 / \xi$. If $x_{2}>x_{1}$ the NMC phase disappears.

In Figs. (14,22) we show the numerical phase space of the model for some parameter values. The plots are obtained integrating Eq. (17) for several initial conditions, and then performing a Poincaré projection onto the unitary circle. For any trajectory we can identify four stages, or cosmological epochs. All trajectories start ideally from the initial singularity at $\phi, \dot{\phi} \rightarrow \infty$, and they fall after a short transient on the inflationary attractors. In this first stage the higher-order terms that we are neglecting in this section can be important. The second stage is represented by the outer part of the attractors, where the $\mu$-terms are dominant; now the trajectories follow Eq. (23), and the cosmic expansion is given by (26). The third stage begins when $x<x_{1}$, where the attractors enter the NMC region. Now the behavior will be as described in the introduction, with the power-law expansion (1) (inflationary if $2 \leq M<2+\sqrt{3}$ ). Finally, the coupling terms become negligible (for $x<x_{2}$ ) and the theory reduces to ordinary gravity. The details of this last stage depend on the specific form of $V(\phi)$ for small $\phi$, but eventually (perhaps after a last quasi-deSitter inflationary episode) the mass term will dominate, and a series of damped oscillations around the potential ground state will occur. A Friedmannian expansion then takes place. The basin of attraction of the attractors extends to almost all of the phase space, and all initial conditions lead to the central Friedmann region. Since the higher-order terms do not introduce new critical points in the model, the qualitative picture given here should be of general validity. Notice that Fig. (2) displays a model with $M=4$, outside the successful range in NMC models, but inflationary in the NMDC model.

\section{Conclusions}

We have shown that nonminimal derivative couplings are an interesting source of new cosmological dynamics. Generally speaking, their presence allows inflationary attractors where otherwise they would be absent. The quite narrow range of parameters that allows a viable inflation in NMC theories is here expanded to a semi-infinite range. Let us remark that the succession of different inflationary epochs may be used to break the scale-invariance of inflationary perturbation spectra21. In the model effectively considered, three inflationary phases (quasi-deSitter, power-law, quasi-deSitter) may occur, depending on the parameter. This realizes a scenario of double (or triple) inflation 21 without the need to introduce more fields.

Before concluding, let us comment on another possible consequence of a NMDC cosmol-

ogy. The inflationary attractors found in the previous section can be used to implement 
a model of primordial phase transition along the scheme of extended inflation. Suppose a second scalar field $\psi$ with double-well self-interaction potential $V_{2}(\psi)$ is present in the theory. During the inflationary stage driven by $\phi$ the new field may perform a first-order phase transition from the more energetic false-vacuum state to the less energetic true vacuum. The transition proceeds through quantum and thermal nucleation of true-vacuum bubbles, whose surface energy density contains the "latent heat" of the process. The bubbles grow converting the background false-vacuum energy density into surface "kinetic energy" and eventually coalesce and percolate, unless the false-vacuum inflationary expansion is too rapid. If $\Gamma$ is the nucleation rate, the (not normalized) probability that a point is still in the false-vacuum state at the time $t$ is 22

$$
p_{F V}(t)=a^{3}(t) e^{-I(t)},
$$

where

$$
I(t)=4 \pi / 3 \int_{0}^{t} d t^{\prime} \Gamma a^{3}\left(t^{\prime}\right)\left[\int_{t^{\prime}}^{t} \frac{d u}{a(u)}\right]^{3},
$$

where $t=0$ is the instant in which the nucleation begins. (Let us remark that $p_{F V}$ should be calculated in an Einstein frame; for what concerns the following discussion this precisation is of secundary importance.) If $p_{F V} \rightarrow 0$ the transition is completed. It is well known that in old inflation, where the expansion is exponential, the transition can never be completed; extended inflation cures this "graceful exit" problem by slowing down the inflation to a power-law. In our model the scale factor expands according to Eq. (26) during the NMDC phase (the second stage) and according to Eq. (1) during the NMC phase (the third stage). It is the latter phase to be crucial. During the quasi-deSitter NMDC expansion the false vacuum grows so fast that the bubbles nucleated are rapidly diluted before they can coalesce, unless $\Gamma$ is unrealistically large. This is the same problem one has in old inflation. However, during the subsequent NMC epoch the false vacuum slows down to a power-law expansion (it does not matter here whether inflationary or not) and the phase transition can find a natural exit, just as one has in extended inflation. One should also ensure that the possible last inflationary phase in the minimally coupled regime, which depends on the details for $|\phi| \rightarrow 0$ of $V(\phi)$, be negligible. In this scenario, the transition occurs almost entirely during the NMC epoch, when the scale factor expands slowly enough. Also the bubble nucleation will take place mostly in the NMC epoch. Depending on the relative duration of the NMDC inflationary phase and the NMC phase one can have different bubble spectra. If the quasideSitter NMDC phase lasts for a large fraction of the 60 or so $e$-foldings one needs for the inflation to be successful, the bubbles produced during the subsequent power-law NMC phase are very small, they rapidly thermalizes (perhaps with a production of gravitational waves 23 ) and do not affect neither the large scale structure, nor the cosmic microwave background 4 . If, on the contrary, the NMC phase takes, say, $55 e$-foldings, then the bubbles would be today very large and hardly thermalized. The cosmic microwave background measurements and the primordial nucleosynthesis would put very stringent limits on the model, but the intriguing possibility that the primordial bubbles do participate in the large-scale structure formation arises 25. 


\section{ACKNOWLEDGMENTS}

The author thanks A. A. Starobinsky, H.-J. Schmidt and G. Pollifrone for thoughtful comments on the manuscript. 


\section{Figure Caption}

1. Poincaré phase space for a model with $m=1, M=3, \xi=\mu=-1, \lambda=3 / 2$. The inflationary attractors are clearly seen. The trajectories start at $t=0$ from the "north" and "south" poles and rapidly reach the attractors. Eventually, all trajectories fall onto the global stability point at $\dot{\phi}=\phi=0$, after a number of damped oscillations. The discrete symmetry $\phi, \dot{\phi} \rightarrow-\phi,-\dot{\phi}$, also evident in the Lagrangian, shows up.

2. Poincaré phase space for a model similar to the precedent, but with $M=4$. In a NMC theory this model would not be inflationary.

\section{References}

1. A. Linde, Phys. Lett. 129B (1983) 177; Nucl. Phys. B216 (1983) 421 (1983).

2. F. Lucchin and S. Matarrese, Phys. Rev. D32 (1985) 1316.

3. R. Wald, Phys. Rev. D28 (1983) 2118; K.-I. Maeda, Phys. Rev. D37 (1988) 858.

4. U. Kasper, Nuovo Cim. B103 (1989) 291; T. Futamase and K. Maeda, Phys. Rev. D39 (1989) 399; L. Amendola, M. Litterio and F. Occhionero, Int. J. Mod. Phys. A 5 (1990) 3861.

5. P. A. M. Dirac, Proc. R. Soc. A338 (1974) 439; P. Jordan, Schwerkraft und Weltall (Wieweg, Braunschweig, 1955); C. Brans and R. H. Dicke Phys. Rev., 124 (1961) 925.

6. N. D. Birrell and P. C. W. Davies, Quantum Fields in Curved Spaces (Cambridge Univ. Press, Cambridge, 1982).

7. A. Zee, Phys. Rev. Lett. , 42 (1979) 417; F. S. Accetta, D. J. Zoller and M. S. Turner, Phys. Rev. D31 (1985) 3046.

8. E. W. Kolb and M. S. Turner, The Early Universe (Addison-Wesley, Menlo Park, CA, 1990).

9. S. Randjbar-Daemi, A. Salam, and J. Strathdee, Phys. Lett. B135 (1984) 388; K. Maeda, Class. Quantum Gravit. 3 (1986) 233; T. Applequist and A. Chodos, Phys. Rev. Lett. 50 (1983) 141.

10. M. Morikawa, Ap. J. Lett. , 362 (1990) L37.

11. J. Yokohama, Phys. Lett., B212 (1988) 273.

12. J. Bekenstein and M. Milgrom, Ap. J., 286 (1984) 7. 
13. D. La and P. J. Steinhardt, Phys. Rev. Lett. , 62 (1989) 376; D. La and P. J. Steinhardt, Phys. Lett. B220 (1989) 375; F. S. Accetta and J. J. Trester, Phys. Rev. D 39 (1989) 2854; A. Linde, CERN-TH-5806/90 preprint (1990).

14. P. J. Steinhardt and F. S. Accetta, Phys. Rev. Lett. 64 (1990) 2740.

15. R. Holman, E. W. Kolb, and Y. Wang, Phys. Rev. Lett. 65 (1990) 17; R. Holman, E. W. Kolb, S. L. Vadas and Y. Wang, Phys. Rev. D43 (1991) 995; D43 (1991) 3833; Y. Wang, Phys. Rev. D 42 (1990) 2541.

16. Y. Wang, Phys. Rev. D42 (1990) 2541; D. La, Phys. Rev. D44 (1991) 1680; L. Amendola, S. Capozziello, M. Litterio and F. Occhionero, Phys. Rev. D45 (1992) 417.

17. T. Damour, G. W. Gibbons and C. Gundlach, Phys. Rev. Lett. 64 (1990) 123; G. Piccinelli, F. Lucchin and S. Matarrese, Phys. Lett. B277 (1992) 58.

18. L. Amendola, D. Bellisai and F. Occhionero, in preparation.

19. B. Whitt, Phys. Lett. 145B (1984) 176; J. D. Barrow and Cotsakis S., Phys. Lett., 214B (1988) 515; K.-I. Maeda, Phys. Rev. D39 (1989) 3159; H.-J. Schmidt, Class. Q. Grav. 7 (1990) 1023.

20. G. Magnano, M. Ferraris and M. Francaviglia, Gen. Relativ. Gravit. , 19 (1987) 465.

21. L. A. Kofman, A. Linde and A. Starobinsky, Phys. Lett. B157 (1985) 361; L. Amendola, F. Occhionero and D. Saez, Ap. J. 349 (1990) 399.

22. A. H. Guth and E. J. Weinberg, Phys. Rev. D23 (1981) 876; Nucl. Phys. B212 (1983) 321.

23. M. S. Turner and F. Wilczek Phys. Rev. Lett. 66 (1991) 5.

24. M. S. Turner, Fermilab-Conf-91/220-A, preprint (1991); M. S. Turner, E. J. Weinberg and L. M. Widrow, Fermilab-Pub-91/334-A, preprint (1992).

25. D. La, Phys. Lett. B265 (1991) 232. 